\title{
Narrating psychological distress: Associations between cross-clausal integration and mental health difficulties
}

\author{
JÖRG ZINKEN \\ University of Portsmouth \\ CAROLINE BLAKEMORE \\ University of Southampton \\ KATARZYNA ZINKEN \\ Warsaw School of Social Sciences and Humanities \\ LISA BUTLER \\ Portsmouth City Primary Care Trust \\ T. CHAS SKINNER \\ Combined Universities Centre for Rural Health
}

Received: May 20, 2008 Accepted for publication: January 24, 2010

\author{
ADDRESS FOR CORRESPONDENCE \\ Jörg Zinken, Department of Psychology, University of Portsmouth, King Henry Building, King \\ Henry I Street, Portsmouth PO1 2DY, UK. E-mail: joerg.zinken@port.ac.uk
}

\begin{abstract}
Psychological research has emphasized the importance of narrative for a person's sense of self. Building a coherent narrative of past events is one objective of psychotherapy. However, in guided self-help therapy the patient has to develop this narrative autonomously. Identifying patients' narrative skills in relation to psychological distress could provide useful information about their suitability for self-help. The aim of this study was to explore whether the syntactic integration of clauses into narrative in texts written by prospective psychotherapy patients was related to mild to moderate psychological distress. Cross-clausal syntax of texts by 97 people who had contacted a primary care mental health service was analyzed. Severity of symptoms associated with mental health difficulties was assessed by a standardized scale (Clinical Outcomes in Routine Evaluation outcome measure). Cross-clausal syntactic integration was negatively correlated with the severity of symptoms. A multiple regression analysis confirmed that the use of simple sentences, finite complement clauses, and coordinated clauses was associated with symptoms $\left(R^{2}=.26\right)$. The results suggest that the analysis of cross-clausal syntax can provide information on patients' narrative skills in relation to distressing events and can therefore provide additional information to support treatment decisions.
\end{abstract}


Guided self-help based on cognitive behavioral therapy has been recommended for the treatment of mild to moderate forms of common mental health difficulties by the British National Institute for Health and Clinical Excellence (2004). When guided self-help is effective, it constitutes an efficient form of mental health service provision (Gellatly et al., 2007). Such efficiency is vital given the long waiting times for mental health care (Marks, 2004).

One difficulty in optimizing efficiency lies in the identification of patients for whom self-help works, as indicated by the high number of dropouts from mental health care programs (e.g., Gellatly et al., 2007; Ghaderi, 2006). Selfreport measures assessing the severity of psychological distress can only tell us whether a patient qualifies for self-help, that is, whether their symptoms can be classified as "mild to moderate." However, they do not give further information about the likelihood that self-help is a suitable form of therapy for this patient.

In the mental health service where the present study was carried out, practitioners have started asking patients for brief free texts about their current and previous problems. This is done in the hope that such texts might give us useful additional information on which to base treatment decisions. The present study is a first attempt to explore the clinical value of these texts.

Our starting point is a set of findings relating to the importance of coherent narratives for a person's sense of self (Bruner, 1991; Gallagher, 2000). We can minimally define narrative as the recapitulation of past experience in language (Labov, 1972, p. 359). In the area of mental health research, qualitative analysis of spontaneous speech has indicated that depressed individuals, when attempting such recapitulation, were "rambling, repetitious, and vague" and had "difficulty in connecting ... topics" (Bucci \& Freedman, 1981, p. 348). ${ }^{1}$ Recovery from psychological distress, in turn, seems to be associated with the development of a coherent "story" about distressing events (Pennebaker \& Seagal, 1999). Research on expressive writing by psychologically distressed individuals has suggested that people gain the most benefits from writing when they increasingly make relations between the narrated events over consecutive writing sessions, as indicated by the use of relational terms such as reason, because, or why (Pennebaker, Mayne, \& Francis, 1997; Pennebaker \& Seagal, 1999). Experimental research in this area has suggested that forming a narrative may be necessary for expressive writing to produce effects (Smyth, True, \& Souto, 2001). Smyth and colleagues (2001) asked participants in one experimental group to write about traumatic events in a list format, whereas participants in a second experimental group were asked to write about such events narratively. Whereas participants in the "list" group did not differ from the control group that had been asked to write about neutral topics, participants in the "narrative" group reported less restriction of activity in relation to illness and higher avoidant thinking than the other two groups over a 5-week period after the intervention.

Against the background of these findings, it seems plausible that for guided selfhelp to work, patients need to be capable of reflecting on distressing events and of writing about these in a narrative format. However, the construct of "narrativity" is difficult to capture precisely. Most studies of relationships between language use and psychological distress have focused on word counts (Pennebaker, Mehl, $\&$ Niederhoffer, 2003). From this perspective, the use of words such as because or 
reason has been interpreted (post hoc) as indicating narrative writing (Pennebaker et al., 1997). This seems reasonable in so far as these words can be used to integrate two statements: of an event and of an explanation. However, the use of word counts is bound to have its limits: surely a person can write in a narrative manner without using any word in particular.

In the present study, we therefore explore whether an analysis of grammar, in particular of cross-clausal syntax, can capture some aspects of narrative. Following Labov's definition of narrative, recapitulation of past experience is achieved "by matching a verbal sequence of clauses to the sequence of events" (Labov, 1972, p. 359). Labov provides an example of a typical preadolescent narrative (p. 360):

(1) a. This boy punched me

b. and I punched him

c. and the teacher came in

d. and stopped the fight.

This short narrative consists of four clauses, sequentially related to adjacent clauses in such a way that they recapitulate the narrated events as they occurred. Clauses can be defined as the smallest possible sentential expression of an event (Croft, 1998), bringing together a subject and a predicate. Specific events are verbalized in so-called "grounded" clauses (Langacker, 1987), that is, in clauses that situate the described event in a particular person's temporal "landscape." Grammatically speaking, a grounded clause contains a finite verb. For example, "I feel alright" is a grounded clause, whereas "Feeling alright" is not. The relevant difference between the two is that "I feel alright" specifies who is feeling alright when.

The present study explored cross-clausal syntax, that is, the ways in which clauses are sequentially put together. Grounded clauses can have a number of relationships to adjacent clauses (Talmy, 2000). Consider the following four minitexts:

(1) (a) I feel alright. (b) I don't wake up at night.

(2) (c) I feel alright, (d) and I don't wake up at night.

(3) (e) When I don't wake up at night, (f) I feel alright.

(4) (g) I think (h) I feel alright, (i) and I don't wake up at night.

No relationship at all is expressed to hold between the two clauses in (1), which constitute two simple sentences. The clause in (2d) is coordinated with the adjacent clause. Such coordination can express a mere coexistence of the two described events, although it would seem that a certain causal relationship between the two can easily be implied. However, grammatically speaking, the clauses in (1) and (2) are independent from one another. The clauses in (3) and (4), in contrast, exhibit hierarchical relationships. Here, (3e) is an adverbial clause, which is subordinate to the main clause, (3f). Adverbial clauses explicitly mark relationships of condition, causation, or temporal demarcation, and thus provide for a very specific narrative integration of two events. Finally, $(4 \mathrm{~h})$ is a complement clause completing the matrix clause "I think." Formally, $(4 \mathrm{~g})$ and $(4 \mathrm{~h})$ exhibit the strongest form of 
integration between two clauses, because the matrix clause would be incomplete without a complement.

The four possible relationships of a "grounded" clause to adjacent clauses are explored in this study: no relationship (simple sentences), coordination (coordinated clauses), subordination (adverbial clauses), and complementation (finite complement clauses).

Simple sentences are the clearest example of lack of narrative integration. A text consisting entirely of isolated simple sentences would not be a narrative, because there would not be any kind of sequential relationship between the events expressed in the individual clauses. We would therefore expect that someone who finds it difficult to narratively capture past experiences would rely on simple sentences to a relatively large extent. Adverbial clauses, which explicitly state the relationship that holds between two events, are the clearest example of narrative integration. We would therefore expect that someone who is capable of narratively capturing past experiences would rely on adverbial clauses to a relatively large extent.

Coordination and complementation are common features of narratives. For example, the narrative by a preadolescent child cited above contains four clauses, three of which are coordinated clauses. However, it seems difficult to make clear predictions about how the use of coordination and complementation might be associated with the overall capability of narratively capturing past experiences. On the one hand, it would seem that a heavy use of coordination can lead to a mere chaining of events with little insight (I feel bad and I can't sleep and I feel tired ...). In contrast, such insights might be afforded by coordination because causal and temporal information is easily available in such constructions. It is only a small step from I feel bad and I can't sleep to the possibly relevant insight I feel bad because I can't sleep. Complementation, in turn, is a special case among the cross-clausal relationships considered here. Clauses brought together by complementation (such as I think I feel alright) do not express two individual events. In relation to the acquisition of complex grammatical structures by children, it has been argued that the matrix clauses of sentences involving complementation (in this case: $I$ think) are best understood not as superordinate clauses, but as evidential markers, giving information about the subject's certainty regarding the main information (in this case: I feel alright; Tomasello, 2007). Sentences such as "I think I feel alright" should then be treated as simple sentence, and we might expect that their use is particularly characteristic of individuals who are finding narrative formation difficult. In contrast, formally speaking, complementation constitutes a hierarchical integration of two clauses, as does subordination with adverbial clauses.

In the following study we explored associations between cross-clausal relationships and psychological distress. Because the work of integrating events in narrative has been shown to be difficult for people experiencing psychological distress, we would expect a negative correlation between narrative integration and psychological distress. If cross-clausal syntax can be shown to be an acceptable approximation toward the broader concept of narrative integration, the analysis of syntax could provide useful information about the current narrative skills of patients considered for self-help. 
Our study assessed the following hypotheses.

Hypothesis 1: There will be a positive correlation between psychological distress and the use of simple sentences, and a negative correlation between psychological distress and the use of adverbial clauses.

Hypothesis 2: There will be a correlation between psychological distress and the use of coordinated clauses, and between psychological distress and the use of complement clauses.

\section{METHOD}

\section{Participants}

The study had a cross-sectional design. Participants had been referred to a Mental Health Primary Care Trust in the south of the United Kingdom because of symptoms associated with mental health difficulties, but were not currently receiving adult mental health services. Data from 100 participants who had been considered for a 10-week guided self-help program were randomly taken from a database of approximately 800 patients who have made contact with the mental health clinic since the guided self-help program was introduced. A sample of 100 participants is appropriate to detect medium size effects with a power of $80 \%$. Three participants' files were empty, so that the data from 97 patients were included in the analysis. All participants gave written consent for their data to be used for quality assurance purposes. The requirement to receive approval for the study from the Local Research Ethics Committee was waived by the Committee because no intervention was used in addition to the one already in use in the clinic; only existing data, routinely collected from all patients, were used; and patients were not allocated to different groups.

Participants' socioeconomic status was estimated based on ACORN classifications of the postcode of participants' places of residence (http://www.upmystreet. com/enter-location/l/?fpage $=\% 2$ Flocal $\% 2 F m y$-neighbours $\% 2 F)$. This classification ascribes each UK postcode a score between 1 (very low) and 5 (very high) for typical family income and educational background.

\section{Clinical Outcomes in Routine Evaluation (CORE) outcome measure}

When first making contact with the mental health clinic, all prospective patients received an information pack from one of the professionals working in the clinic. Patients were asked to complete the documents in this information pack at home and to return it if they were interested in the service that is offered in this clinic. As part of this information pack, all participants completed the CORE outcome measure, a brief self-report questionnaire that is used to evaluate the effectiveness of psychological therapy by assessing psychological distress on four CORE subscales: well-being, perceived problems, functioning, and risk behaviors (Evans et al., 2002). The CORE consists of 34 items, all of which are answered using a 5-point scale ranging from not at all to all the time. Higher scores indicate more severe problems. All four subscales of the CORE have been shown to have 
appropriate internal reliability $(\alpha>0.75$ and $<0.95)$ in clinical and nonclinical samples. Test-retest stability has been shown to be excellent (0.87-0.91) for the well-being, perceived problems, and functioning subscales, and somewhat lower (0.64) for the risk subscale (Evans et al., 2002).

\section{Syntactic structure}

As part of the information pack, prospective patients were also asked to write brief free texts about current and previous problems. The information packs contained a nearly blank sheet with minimal instructions: "Current problems" was written at the top of the sheet, and "Previous problems" was written in the middle of the sheet. There were no further instructions, for example, concerning the content or length of the required text, or the time frame within which to write it. Patients wrote these texts at a place of their choice and handed them in, together with the completed CORE questionnaire, in their own time. The average length of texts included in the present study was 77 words $(S D=49)$, ranging from a minimum of five to a maximum of 385 words.

The texts were parsed into clauses, and clauses were coded manually to investigate the narrative integration of texts. Each clause was coded into one of eight comprehensive and mutually exclusive categories, depending on the presence and type of relationship to neighboring clauses. The coding manual is available from the first author. The types of clauses relevant for the present analysis were "grounded" clauses, that is, those in which the writer had made an effort to verbalize a specific event. This criterion was satisfied by grammatical clauses containing a finite verb: simple sentences, finite complement clauses, coordinated clauses, and adverbial clauses.

Simple sentences (such as "I feel tired. $\#^{2} I^{\prime}$ 'm constantly worried") are complete grammatical sentences existing of only one clause, with no indication of a relationship of the verbalized event to other events. Finite complement clauses (such as "I know \# I have a serious illness") are integrated with a matrix clause, which could not function as the verbalization of an event on its own. Coordinated clauses (such as "I feel tired, \# and I'm constantly worried") relate one verbalized event to another event, but the relationship is very general, for example, coexistence of the two events in the example. Adverbial clauses involve the expression of a more specific relationship, for example, of causality, between the events verbalized in the adverbial clause and the matrix clause (such as "I feel tired, \# because I'm constantly worried").

\section{Reliability of the syntax coding}

Clauses were initially coded by a psychology student with no prior training in syntax, and the coding was then checked and corrected by the first author. Both coders were blind to participants' CORE scores. It is possible to correctly code all clauses on the basis of objective criteria described in the linguistic literature on syntax. Tallerman's (2005) introductory textbook was used as the final arbiter on coding decisions in the present project. We calculated interrater agreement in order to get an idea of how easy (or difficult) the syntax coding is for a novice. 
After three rounds of coding and discussion, the overall percentage of agreement was $85 \%$, corresponding to a Cohen $\mathrm{K}$ value of 0.82 . Individually, percentage of agreement was satisfactory for the variables simple sentences $(85 \%)$, coordinated clauses $(81 \%)$, and adverbial clauses (100\%). Agreement was markedly lower for complement clauses ( $43 \%$ ). These clauses, which do not form potentially complete sentences, are apparently more difficult to identify for coders without linguistic training. Coding decisions were made using Tallerman (2005).

\section{Statistical analysis}

Pearson product-moment correlation coefficients were calculated to assess associations between the structure of texts and the severity of psychological distress. Syntactic variables that were found to be significantly correlated with CORE scores were employed in a regression analysis to further explore associations between language and psychological distress. Statistical analyses to evaluate Hypothesis 1 were one tailed, analyses to evaluate Hypothesis 2 were two tailed. All analyses were performed using the Statistical Package for the Social Sciences (SPSS), version 14 for Windows.

\section{RESULTS}

Descriptive statistics on the sample are provided in Table 1. A score of syntactic integration was calculated by subtracting the percentage of simple sentences, as the clearest case of a lack of narrative integration, from the percentage of adverbial clauses, as the clearest case of narrative integration across clauses. ${ }^{3}$ The possible score for syntactic integration therefore ranges from -100 to 100 .

Cross-clausal syntactic integration showed a significant negative correlation with all CORE subscales apart from the risk subscale (Table 2).

Simple sentences, finite complement clauses, and coordinated clauses showed the expected positive correlation with the severity of psychological distress. Adverbial clauses were not significantly correlated with the severity of psychological distress. Table 3 presents a syntactically simple text fragment written by a patient with a relatively high CORE score, and a syntactically more integrated text fragment written by a patient with a relatively low CORE score. There were no significant correlations between measures of syntactic integration and socioeconomic variables (family income and education, all $p \mathrm{~s}>.4$ ).

We further explored associations between syntactic integration and psychological distress by using syntactic variables that were significantly correlated with overall CORE scores (simple sentences, finite complement clauses, and coordinated clauses) in a regression analysis. All variables were retained in a regression analysis using a backward procedure (see Table 4), which means that simple sentences, complement clauses, and coordinated clauses were each associated with psychological distress independently of the other variables. Syntactic integration accounted for $26 \%$ of the variance in the severity of psychological distress as assessed by the CORE scales. The assumption of no multicollinearity between syntactic variables was met (average variance inflation factor $=1.03$, average tolerance $=0.97$ ) 
Table 1. Descriptive statistics on the sample $(N=97,69$ male $)$

\begin{tabular}{lrr}
\hline \hline & Mean & \multicolumn{1}{c}{$S D$} \\
\hline Age & 39.02 & 12.80 \\
Socioeconomic status & \\
$\quad$ Family income & & \\
$\quad$ Education to a degree $_{\text {CORE score }}{ }^{b}$ & 2.67 & 0.76 \\
Well-being & 2.80 & 1.19 \\
Perceived problems & 1.98 & 0.72 \\
Functioning & 2.46 & 0.87 \\
Risk behavior & 2.47 & 0.83 \\
Syntactic integration & 2.01 & 0.81 \\
Simple sentences & 0.53 & 0.67 \\
Finite complement clauses & -0.97 & 13.50 \\
Coordinated clauses & 8.12 & 10.92 \\
Adverbial clauses & 5.72 & 7.44 \\
\hline \hline
\end{tabular}

${ }^{a}$ Based on the ACORN database, scores ranged from 1 (very low) to 5 (very high). Simple sentences, finite complement clauses, coordinated clauses, and adverbial clauses are shown as mean percentage of clauses out of the total number of clauses used in the text.

${ }^{b} \mathrm{CORE}$ scores ranged from 1 to 5 , with higher scores indicating greater psychological distress. In a previous study (Evans et al., 2002), the mean scores of a nonclinical sample $(n=1084)$ were well-being $=0.91$, perceived problems $=0.90$, functioning $=0.85$, and risk $=0.20$. The mean scores of a clinical sample $(n=863)$ were wellbeing $=2.37$, perceived problems $=2.31$, functioning $=$ 1.86 , and risk $=0.63$. The cutoff scores between clinical and nonclinical populations are well-being $=1.37$ for male and 1.77 for female; perceived problems $=1.44$ for male and 1.62 for female; functioning $=1.29$ for male and 1.30 for female; and risk $=0.43$ for male and 0.30 for female.

\section{DISCUSSION}

In this study, we found that the syntactic integration of clauses in texts written by patients with symptoms of psychological distress at the time of the intake assessment correlated with the severity of their symptoms as assessed by a standardized scale (CORE outcome measure). Participants with stronger symptoms associated with mental health difficulties used relatively more isolated clauses (simple sentence). However, the prediction that such participants would use significantly fewer adverbial clauses was not supported. There was also a significant positive correlation between the coordination of clauses and psychological distress, as well as between the use of complementation and psychological distress. 
Table 2. Correlations between CORE scores and cross-clausal syntax

\begin{tabular}{|c|c|c|c|c|c|}
\hline & \multirow[b]{2}{*}{$\begin{array}{c}\text { Syntactic } \\
\text { Integration }\end{array}$} & \multirow[b]{2}{*}{$\begin{array}{c}\text { Simple } \\
\text { Sentences }\end{array}$} & \multicolumn{3}{|c|}{ Clauses } \\
\hline & & & Adverbial & $\begin{array}{c}\text { Finite } \\
\text { Complement }\end{array}$ & Coordinated \\
\hline CORE score & $-.216^{*}$ & $.225^{*}$ & -.053 & $.333 * *$ & $.287 * *$ \\
\hline Well-being & $-.193^{*}$ & .205 & -.043 & $.292 * *$ & $.238^{*}$ \\
\hline Perceived problems & $-.267 * *$ & $.244^{*}$ & -.110 & $.224 * *$ & $.206^{*}$ \\
\hline Functioning & $-.231 *$ & $.245^{*}$ & -.051 & $.354^{* *}$ & $.310^{* * *}$ \\
\hline Risk behavior & -.049 & .113 & .069 & $.330 * *$ & .202 \\
\hline
\end{tabular}

$* p<.05 . * * p<.01$. Significance levels for syntactic integration, simple sentences, and adverbial clauses are one tailed. Significance levels for coordinated clauses and finite complement clauses are two tailed.

Table 3. Texts with high and low syntactic complexity

\begin{tabular}{llll}
\hline \hline \multicolumn{2}{c}{ High Syntactic Complexity ${ }^{a}$} & & Low Syntactic Complexity $^{b}$ \\
\hline $\mathrm{K}$ & Anxiety & SS & I find my job very stressful. \\
$\mathrm{K}$ & Panic attacks & $\mathrm{MC}$ & I feel \\
$\mathrm{K}$ & Depression & FC & I am not coping. \\
$\mathrm{MC}$ & I'm managing. & $\mathrm{CC}$ & Am constantly worrying and paranoid \\
$\mathrm{NFC}$ & To go to work & $\mathrm{MC}$ & I moved to X in September last year. \\
$\mathrm{AC}$ & Because I am trying & $\mathrm{CC}$ & And find \\
$\mathrm{NFC}$ & To keep my routine as normal \\
& FC & I still do not know many people. \\
$\mathrm{MC}$ & I find it difficult at home. & $\mathrm{MC}$ & I am constantly worrying about \\
$\mathrm{AC}$ & Because my mind is not & $\mathrm{CC}$ & work money etc. \\
& $\quad$ so occupied & & \\
\hline \hline
\end{tabular}

Note: K, keyword clause; MC, main clause; NFC, nonfinite complement; AC, adverbial clause; $\mathrm{CC}$, coordinated clause; FC, finite complement; SS, simple sentence.

${ }^{a}$ Author's CORE score $=1.59$.

${ }^{b}$ Author's CORE score $=2.5$.

Table 4. Contribution of syntactic integration to the prediction of severity of psychological distress

\begin{tabular}{lccccr}
\hline \hline & $B$ & $S E B$ & $\beta$ & \multicolumn{2}{c}{$95 \%$ CI for $B$} \\
\hline Constant & 1.40 & 0.12 & & 1.15 & 1.64 \\
Simple sentences & 0.02 & 0.01 & $0.30^{* * *}$ & 0.01 & 0.03 \\
Finite complement clauses & 0.03 & 0.01 & $0.33^{* *}$ & 0.01 & 0.05 \\
Coordinated clauses & 0.02 & 0.01 & $0.30^{* *}$ & 0.01 & 0.03 \\
\hline \hline
\end{tabular}

Note: $R^{2}=.26$.

${ }^{* *} p<.01$. 
The results suggest that the analysis of cross-clausal syntax might be a useful way of studying current skills of patients considered for guided self-help to narratively recapitulate past experiences. Despite the recent interest in language in the context of mental health (see Sloan \& Marx, 2004), this is, to the best of our knowledge, the first study in this field that quantitatively investigates texts at the very concrete and objective level of syntactic structure.

An open question concerns the relationship between the syntactic structure and the content of texts. In this study, the use of finite complement clauses (such as "I know \# that I can't change it") was most strongly correlated with selfreport measures of psychological distress. One possible explanation is that such constructions should be considered as simple sentences, and not as a hierarchical integration of two clauses (Tomasello, 2007). It is also possible that this syntactic structure is mainly used to express a particular type of content. Matrix clauses often used with finite complement clauses are I think, I feel, and I know, which express a reflective, "inward" perspective. Clearly, both the lexical dimension and the syntactic dimension of the narrative need to be studied for a full picture of the relations between language use and mental health.

Overall, the texts analyzed in this study showed little syntactic integration (see Table 1). This bias toward a production of simple texts was to some extent encouraged by the writing instructions and the materials provided. There was little space for participants to write about their current and previous problems (one page), and the writing instructions were formulated as keywords ("current problems," "previous problems"). Formulating the writing instructions in grammatical sentences, and providing more space might encourage participants to write more. This might further increase the diagnostic value of the texts. It is noteworthy that the correlation of adverbial clauses with the severity of psychological distress, although not significant, pointed in the expected, negative, direction. Future research could evaluate whether longer texts with more overall syntactic integration show this correlation more clearly.

Although psychiatric services informed by cognitive behavioral therapy have recognized the need to help patients in restructuring their thinking, recent research has highlighted the possibility that it might be beneficial for people experiencing mental health difficulties to develop a structured narrative in the first place (Pennebaker et al., 1997; Pennebaker \& Seagal, 1999). The results of the current study suggest that cross-clausal integration might be an acceptable approximation toward the broader, and more difficult to capture, construct of narrativity. If this is the case, it would be possible to offer participants of guided self-help programs very concrete guidance on how they can make their writing more "narrative."

Overall, the present study suggests that the syntactic analysis of free texts can provide additional information, beside that gained from psychometric questionnaires, to inform treatment decisions. In the activity of writing free texts, patients gave us an insight into how they "work" in a guided self-help context, that is, their ability and motivation to reflect on their situation. A patient reporting mild to moderate symptoms of mental health difficulties who narratively integrates verbalized events using adverbial clauses, and largely avoids the use of isolated, simple sentences, might be in the right position to gain benefits from guided self-help. A patient reporting mild to moderate symptoms of mental health difficulties who 
largely relies on isolated, simple sentences might be better served with a different form of therapy. Associations between the syntactic integration of clauses in texts written at the time of the intake assessment and outcomes of guided self-help will be the focus of our next study.

In the present study, we coded clauses manually. This is both time consuming and requires some linguistic expertise, both of which make this impracticable for clinical practice. However, the computerized parsing and coding of crossclausal syntax, although currently unavailable, is within the reach of contemporary computational linguistics (McEnery, Tono, \& Xiao, 2006). If further research bears out the existence of reliable associations between cross-clausal syntax and aspects of mental health, this could be a worthwhile future interdisciplinary project.

\section{ACKNOWLEDGMENTS}

We gratefully acknowledge the detailed comments by two anonymous reviewers on an earlier version of this paper.

\section{NOTES}

1. To the best of our knowledge, there is no research investigating language use after an experimental manipulation of mood state.

2. The hash sign indicates a clause boundary.

3. Coordinated and complement clauses have not been taken into account in the calculation of a syntactic integration score. Although both might be located between simple sentences and adverbial clauses in terms of the narrative integration that their use embodies, it currently seems impossible to meaningfully "weight" these differences for the current purpose.

\section{REFERENCES}

Bruner, J. (1991). The narrative construction of reality. Critical Inquiry, 18, 1-21.

Bucci, W., \& Freedman, N. (1981). The language of depression. Bulletin of the Menninger Clinic, 45 , $334-358$.

Croft, W. (1998). The structure of events and the structure of language. In M. Tomasello (Ed.), The new psychology of language. Cognitive and functional approaches to language structure (pp. 67-92). Mahwah, NJ: Erlbaum.

Evans, C., Connell, J., Barkham, M., Margison, F., McGrath, G., Mellor-Clark, J., et al. (2002). Towards a standardised brief outcome measure: Psychometric properties and utility of the CORE-OM. British Journal of Psychiatry, 180, 51-60.

Gallagher, S. (2000). Philosophical conceptions of the self: Implications for cognitive science. Trends in Cognitive Sciences, 4, 14-21.

Gellatly, J., Bower, P., Hennessy, S., Richards, D., Gilbody, S., \& Lovell, K. (2007). What makes self-help interventions effective in the management of depressive symptoms? Meta-analysis and meta-regression. Psychological Medicine, 37, 1217-1228.

Ghaderi, A. (2006). Attrition and outcome in self-help treatment for bulimia nervosa and binge eating disorder: A constructive replication. Eating Behaviors, 7, 300-308.

Labov, W. (1972). The transformation of experience in narrative syntax. Language in the inner city: Studies in the Black English vernacular. Philadelphia, PA: University of Pennsylvania Press.

Langacker, R. W. (1987). Foundations of cognitive grammar: Theoretical prerequisites (Vol. 1). Stanford, CA: Stanford University Press. 
Marks, I. (2004). Psychiatry in the future. Psychiatric Bulletin, 28, 319-320.

McEnery, T., Tono, Y., \& Xiao, R. Z. (2006). Corpus-based language studies: An advanced resource book. London: Routledge.

National Institute for Clinical Excellence. (2004). Depression: Management of depression in primary and secondary care. London: Author.

Pennebaker, J. W., Mayne, T. J., \& Francis, M.E. (1997). Linguistic predictors of adaptive bereavement. Journal of Personality and Social Psychology, 72, 863-871.

Pennebaker, J. W., Mehl, M. R., \& Niederhoffer, K. G. (2003). Psychological aspects of natural language use. Our words, our selves. Annual Review of Psychology, 54, 547-577.

Pennebaker, J. W., \& Seagal, J. D. (1999). Forming a story: The health benefits of narrative. Journal of Clinical Psychology, 55, 1243-1254.

Sloan, D. M., \& Marx, B. P. (2004). Taking pen to hand: Evaluating theories underlying the written disclosure paradigm. Clinical Psychology: Science and Practice, 11, 121-137.

Smyth, J. M., True, N., \& Souto, J. (2001). Effects of writing about traumatic experiences: The necessity for narrative structuring. Joumal of Social and Clinical Psychology, 20, 161-172.

Tallerman, M. (2005). Understanding syntax (2nd ed.). London: Hodder Arnold.

Talmy, L. (2000). Toward a cognitive semantics. Cambridge, MA: MIT Press.

Tomasello, M. (2007). A usage-based approach to child language acquisition. In V. Evans, B. Bergen, \& J. Zinken (Eds.), The cognitive linguistics reader (pp. 834-848). London: Equinox. 